\title{
SOME RESULTS ON GENERALISED VOIGT FUNCTIONS
}

\author{
K. C. GUPTA ${ }^{1}$, S. P. GOYAL ${ }^{2}$ and ROHIT MUKHERJEE ${ }^{2}$
}

(Received 9 February, 1999; revised 18 May, 1999)

\begin{abstract}
Recently, Srivastava, Pathan and Kamanujjama established several results for generalised Voigt functions which play an important role in several diverse fields of physics-such as astrophysical spectroscopy and the theory of neutron reactions. In the present paper we aim to generalise some partly bilateral and partly unilateral representations and generating functions of Srivastava $e t$ al. by considering a specialised version of the Srivastava-Chen definition of the unified Voigt functions. Several special cases of our main results are mentioned briefly.
\end{abstract}

\section{Introduction}

Srivastava and Chen $[2$, p. $71,(41)]$ have provided unified presentations of the Voigt functions $K(x, y)$ and $L(x, y)$ in the following form:

$$
\begin{aligned}
S_{Q: p, q ; u, v ; \zeta}^{P: m, n ;, s, \nu}\left[\begin{array}{l}
\left(\xi_{j} ; e_{j}, f_{j}\right)_{1, P}:\left(\alpha_{j}, A_{j}\right)_{1, p} ;\left(\gamma_{j}, C_{j}\right)_{1, u} ; \\
\left(\eta_{j} ; g_{j}, h_{j}\right)_{1, Q}:\left(\beta_{j}, B_{j}\right)_{1, q} ;\left(\delta_{j}, D_{j}\right)_{1, v} ; x, y
\end{array}\right] \\
=\frac{1}{\sqrt{\pi}}(x / 2)^{v+1 / 2} \int_{0}^{\infty} t^{\zeta+v} e^{-t^{2} / 4} \\
\quad \times H_{P, Q: p, q ; u, v}^{0,0: m, n ; r, s}\left[\begin{array}{l|l}
\frac{1}{4} x^{2} t^{2} & \begin{array}{l}
\left(\xi_{j} ; e_{j}, f_{j}\right)_{1, P}:\left(\alpha_{j}, A_{j}\right)_{1, p} ;\left(\gamma_{j}, C_{j}\right)_{1, u} \\
\frac{1}{4} y^{2} t^{2}
\end{array} \\
\left(\eta_{j} ; g_{j}, h_{j}\right)_{1, Q}:\left(\beta_{j}, B_{j}\right)_{1, q} ;\left(\delta_{j}, D_{j}\right)_{1, v}
\end{array}\right] d t
\end{aligned}
$$

where $H\left[\begin{array}{l}x \\ y\end{array}\right]$ stands for the $H$-function of two variables (see, for example, $[4$, p. 82, (6.1.1)]).

In this paper we aim to generalise the generating function part of the work of

\footnotetext{
'Emeritus Fellow, Department of Mathematics, M. R. Engineering College, Jaipur 302017, Rajasthan, India; e-mail: kcgupta_in2000@yahoo.com.

${ }^{2}$ Department of Mathematics, University of Rajasthan, Jaipur 302004, Rajasthan, India.

(C) Australian Mathematical Society 2002, Serial-fee code 1446-1811/02
} 
Srivastava et al. [5] by considering the following special case of (1):

$$
V_{\eta, \sigma, c}^{\rho, p, q}[x, y, z]=x^{\sigma} \int_{0}^{\infty} t^{\eta} e^{-y t-z t^{c}}{ }_{p} \psi_{q}\left[\begin{array}{l}
\left(a_{j}, \alpha_{j}\right)_{1, p} ; \\
\left(b_{j}, \beta_{j}\right)_{1, q} ;
\end{array}-(x t)^{\rho}\right] d t
$$

where $c, \rho, \sigma, x, y, z, \alpha_{j}, \beta_{j} \in R^{+},(i=1,2, \ldots, p, j=1,2, \ldots, q) ; \operatorname{Re}(\eta)>-1$; $A=1+\sum_{j=1}^{q} \beta_{j}-\sum_{j=1}^{p} \alpha_{j}>0$ and $\psi_{q}$ stands for the Fox-Wright function ([6]).

We shall require the following explicit representation for the generalised Voigt function defined by (2):

$$
\begin{aligned}
& V_{\eta, \sigma, c}^{\rho, p, q}[x, y, z] \\
& =x^{\sigma} z^{-(\eta+1) / c} \frac{\prod_{j=1}^{p} \Gamma\left(a_{j}\right)}{c \prod_{j=1}^{p} \Gamma\left(b_{j}\right)}\left\{\Gamma\left(\frac{\eta+1}{c}\right)\right. \\
& \times F^{(2)}\left[\begin{array}{c}
\left(\frac{\eta+1}{c} ; \frac{\rho}{c}, \frac{2}{c}\right):\left(a_{j}, \alpha_{j}\right)_{1, p} ;-; \\
\left.:\left(b_{j}, \beta_{j}\right)_{1, q} ;\left(\frac{1}{2}, 1\right) ;-x^{\rho} z^{-\rho / c}, \frac{y^{2}}{4} z^{-2 / c}\right]
\end{array}\right. \\
& -y z^{-1 / c} \Gamma\left(\frac{\eta+2}{c}\right)
\end{aligned}
$$

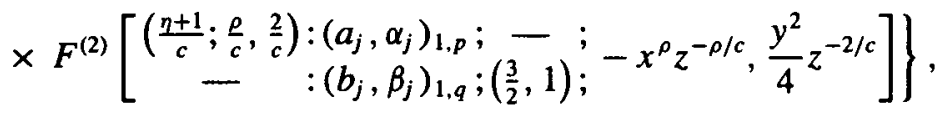

$\left\{c, \rho, \sigma, x, y, z, \alpha_{j}, \beta_{j} \in R^{+}, i=1, \ldots, p, j=1, \ldots, q ; \operatorname{Re}(\eta)>-1 ; A>0\right\}$, where $F^{(2)}$ is a special case of the Srivastava-Daoust function [3, p. 454] for $r=2$.

The above result can be easily proved by following a method similar to that applied by Srivastava et al. for obtaining explicit representations for their generalised Voigt function $[5$, p. $55,(2.4)]$.

\section{Partly bilateral and partly unilateral representations}

We start with a known result due to Pathan and Yasmeen [1] in the following form:

$$
\exp \left(s+t-\frac{x t}{s}\right)=\sum_{m=-\infty}^{\infty} \sum_{n=m^{*}}^{\infty} \frac{s^{m}}{m !} \frac{t^{n}}{n !}, F_{1}[-n ; m+1 ; x]
$$

(where $m^{*}=\max \{0,-m\}$ ), replace $s, t$ and $x$ by $s \xi^{c}, t \xi^{c}$ and $x \xi^{c}$, respectively, and then multiply both sides of (4) by

$$
\xi^{\eta} \exp \left(-v \xi-w \xi^{c}\right)_{p} \psi_{q}\left[\begin{array}{ll}
\left(a_{j}, \alpha_{j}\right)_{1, p} ; \\
\left(b_{j}, \beta_{j}\right)_{1, q} ;
\end{array}-(u \xi)^{\rho}\right] \quad(w>0) .
$$


Applying the technique used by Srivastava et al. $[5$, p. 59, (3.5)] mutatis mutandis, we easily arrive at the following result:

$$
\begin{aligned}
& V_{\eta, \sigma, c}^{\rho, p, q}[u, v, w-s-t+(x t) / s] \\
& =\frac{u^{\sigma} w^{-(\eta+1) / c}}{c} \sum_{m=-\infty}^{\infty} \sum_{n=m^{*}}^{\infty} \frac{(s / w)^{m}}{m !} \frac{(t / w)^{n}}{n !} \sum_{k, l=0}^{\infty} \frac{\prod_{j=1}^{p} \Gamma\left(a_{j}+l \alpha_{j}\right)}{\prod_{j=1}^{q} \Gamma\left(b_{j}+l \beta_{j}\right) l ! k !} \\
& \times\left(-u^{\rho} w^{-\rho / c}\right)^{l}\left(-v w^{-1 / c}\right)^{k} \Gamma\left[\frac{\eta+c m+c n+\rho l+k+1}{c}\right] \\
& \times{ }_{2} F_{1}\left[\begin{array}{cc}
-n,(\eta+c m+c n+\rho l+k+1) / c ; & \frac{x}{w} \\
m+1 ; & ,
\end{array}\right.
\end{aligned}
$$

$\operatorname{Re}(\eta)>-1 ; c, \rho, \sigma, u, v, w, \alpha_{j}, \beta_{j} \in R^{+}, i=1, \ldots, p, j=1, \ldots, q ; A>0$, $\operatorname{Re}(w-s-t+(x t) / s)>0$.

Now separating the $k$-series into its even and odd terms, we obtain

$$
\begin{aligned}
& V_{\eta, \sigma, c}^{\rho, p, q}[u, v, w-s-t+(x t) / s] \\
& =\frac{u^{\sigma} w^{-(\eta+1) / c} \prod_{j=1}^{p} \Gamma\left(a_{j}\right)}{c \prod_{j=1}^{q} \Gamma\left(b_{j}\right)} \sum_{m=-\infty}^{\infty} \sum_{n=m^{*}}^{\infty} \frac{(s / w)^{m}}{m !} \frac{(t / w)^{n}}{n !} \\
& \quad \times\left\{\Gamma ( \frac { \eta + c m + c n + 1 } { c } ) F ^ { ( 3 ) } \left[\begin{array}{c}
\left(\frac{\eta+c m+c n+1}{c} ; \frac{\rho}{c}, \frac{2}{c}, 1\right):\left(a_{j}, \alpha_{j}\right)_{1, p} ; \\
:\left(b_{j}, \beta_{j}\right)_{1, q} ;
\end{array}\right.\right. \\
& \left.-\quad ;(-n, 1) ;-(u)^{\rho} w^{-\rho / c}, \frac{v^{2}}{4} w^{-2 / c}, \frac{x}{w}\right] \\
& \quad\left(\frac{1}{2}, 1\right) ;(m+1,1) ; \\
& -v w^{-1 / c} \Gamma\left(\frac{\eta+c m+c n+2}{c}\right) F^{(3)}\left[\frac{\left(\frac{\eta+c m+c n+2}{c} ; \frac{\rho}{c}, \frac{2}{c}, 1\right):\left(a_{j}, \alpha_{j}\right)_{1, p} ;:\left(b_{j}, \beta_{j}\right)_{1, q} ;}{\left.\left.-(-n, 1) ;-(u)^{\rho} w^{-\rho / c}, \frac{v^{2}}{4} w^{-2 / c}, \frac{x}{w}\right]\right\},}\right.
\end{aligned}
$$

$\operatorname{Re}(\eta)>-1 ; c, \rho, \sigma, u, v, w, \alpha_{i}, \beta_{j} \in R^{+}, i=1, \ldots, p, j=1, \ldots, q ; A>0$, $\operatorname{Re}(w-s-t+(x t) / s)>0$, where $F^{(3)}$ is a special case of the Srivastava-Daoust function [3, p. 454] for $r=3$.

Obviously the result due to Srivastava et al. $[5$, p. 59, (3.6)] is contained in our result (6) as a special case.

When $s=t=x / 2$, (6) gives the partly bilateral and partly unilateral representation of the generalised Voigt function defined by (2). Analogous representations for Voigt functions and their various generalizations introduced in the literature from time to time can be easily obtained from (6) by simply specializing the various parameters therein. 


\section{A set of generating functions}

On expanding the left member of (6) and using the representation (3), we obtain, using the notation $\hat{w}=w-s-t+x t / s$,

$$
\begin{aligned}
& \hat{w}^{-a}\left\{\Gamma(a) F^{(2)}\left[\begin{array}{c}
\left(a ; \frac{\rho}{c}, \frac{2}{c}\right):\left(a_{j}, \alpha_{j}\right)_{1, p} ;-;-u^{\rho} \hat{w}^{-\rho / c}, \frac{v^{2}}{4} \hat{w}^{-2 / c} \\
:\left(b_{j}, \beta_{j}\right)_{1, q} ;\left(\frac{1}{2}, 1\right) ;-u^{2}
\end{array}\right]\right. \\
& -v \hat{w}^{-1 / c} \Gamma\left(a+\frac{1}{c}\right)
\end{aligned}
$$

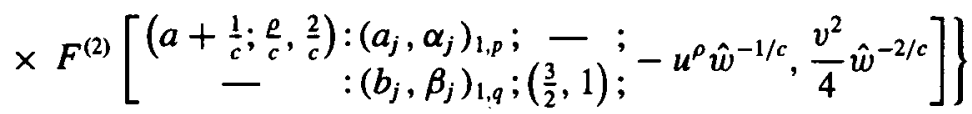

$$
\begin{aligned}
& =w^{-a} \sum_{m=-\infty}^{\infty} \sum_{n=m^{*}}^{\infty} \frac{(s / w)^{m}}{m !} \frac{(t / w)^{n}}{n !}\left\{\Gamma ( a + m + n ) F ^ { ( 3 ) } \left[\begin{array}{c}
\left(a+m+n ; \frac{e}{c}, \frac{2}{c}, 1\right): \\
-
\end{array}\right.\right. \\
& \begin{array}{l}
\left.\left(a_{j}, \alpha_{j}\right)_{1, p} ;-1(-n, 1) ;-u^{\rho} w^{-\rho / c}, \frac{v^{2}}{4} w^{-2 / c}, \frac{x}{w}\right] \\
\left(b_{j}, \beta_{j}\right)_{1, q} ;\left(\frac{1}{2}, 1\right) ;(m+1,1) ;
\end{array} \\
& -v w^{-1 / c} \Gamma\left(a+\frac{1}{c}+m+n\right) F^{(3)}\left[\begin{array}{c}
\left(a+\frac{1}{c}+m+n ; \frac{\rho}{c}, \frac{2}{c}, 1\right): \\
-
\end{array}\right. \\
& \begin{array}{l}
\left.\left.\left(a_{j}, \alpha_{j}\right)_{1, p} ;-;(-n, 1) ;-u^{\rho} w^{-\rho / c}, \frac{v^{2}}{4} w^{-2 / c}, \frac{x}{w}\right]\right\}, \\
\left(b_{j}, \beta_{j}\right)_{1, q} ;\left(\frac{3}{2}, 1\right) ;(m+1,1) ;
\end{array}
\end{aligned}
$$

where $a=(\eta+1) / c$ and $\rho, c, u, v, w, \alpha_{i}, \beta_{j} \in R^{+}, i=1, \ldots, p, j=1, \ldots, q$; $\operatorname{Re} a>0 ; A>0 ; \operatorname{Re} \hat{w}>0$.

For $\rho=c=2$ and $\alpha_{i}=\beta_{j}=1(i=1, \ldots, p, j=1, \ldots, q)(7)$ takes the following form:

$$
\begin{aligned}
& \hat{w}^{-(\eta+1) / 2}\left\{\Gamma\left(\frac{\eta+1}{2}\right) F^{(2)}\left[\begin{array}{l}
\frac{\eta+1}{2}:\left(a_{p}\right) ;-; \\
\left.-\left(b_{q}\right) ; \frac{1}{2} ;-\frac{u^{2}}{\hat{w}}, \frac{v^{2}}{4 \hat{w}}\right]
\end{array}\right]\right. \\
& \left.-\frac{v \Gamma\left(\frac{\eta+2}{2}\right)}{\sqrt{\hat{w}}} F^{(2)}\left[\frac{\frac{\eta+2}{2}:\left(a_{p}\right) ;-;}{-}:\left(b_{q}\right) ; \frac{3}{2} ;-\frac{u^{2}}{\hat{w}}, \frac{v^{2}}{4 \hat{w}}\right]\right\} \\
& =w^{-(n+1) / 2} \sum_{m=-\infty}^{\infty} \sum_{n=m^{*}}^{\infty} \frac{(s / w)^{m}}{m !} \frac{(t / w)^{n}}{n !}\left\{\Gamma\left(\frac{\eta+1}{2}+m+n\right)\right.
\end{aligned}
$$

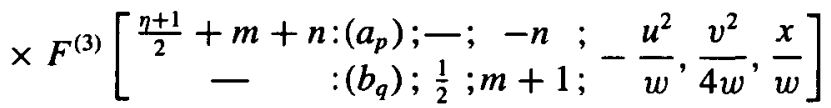

$$
\begin{aligned}
& -\frac{v}{\sqrt{w}} \Gamma\left(\frac{\eta+2}{2}+m+n\right)
\end{aligned}
$$

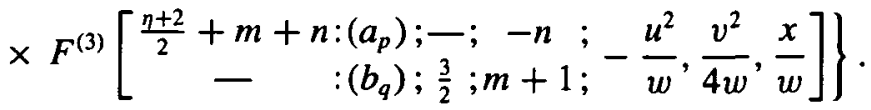


Special cases of (7) and ( 8 ) by setting $u=v=0$ for example can also be cited but we do not record them here for lack of space.

It is not out of place to mention that Equations (4.1)-(4.4) established by Srivastava, Pathan and Kamarujjama [5, pp. 62-63] can be easily obtained as special cases of our results.

\section{Acknowledgements}

The authors are thankful to Professor H. M. Srivastava, University of Victoria, for his valuable suggestions and kind help during the preparation of the paper. The first and second authors (K. C. G. and S. P. G.) are also thankful to the University Grants Commission, New Delhi, for providing some financial assistance for the present work.

\section{References}

[1] M. A. Pathan and Yasmeen, "On partly bilateral and partly unilateral generating functions", J. Austral. Math. Soc. Ser. B 28 (1986) 240-245.

[2] H. M. Srivastava and M. P. Chen, "Some unified presentations of the Voigt functions", Astrophys. Space Sci. 192 (1992) 63-74.

[3] H. M. Srivastava and M. C. Daoust, "Certain generalized Neumann expansions associated with the Kampé de Fériet function", Nederl. Akad. Wetensch. Proc. Ser. A $72=$ Indag. Math. 31 (1969) 449-457.

[4] H. M. Srivastava, K. C. Gupta and S. P. Goyal, The H-functions of one and two variables with applications, (South Asian Publishers, New Delhi, 1982).

[5] H. M. Srivastava, M. A. Pathan and M. Kamarujjama, "Some unified presentations of the generalized Voigt functions", Commun. Appl. Anal. 2 (1998) 49-64.

[6] E. M. Wright, "The asymptotic expansions of the generalized hypergeometric function", J. London Math.Soc. 10 (1935) 286-293. 